\title{
Update Medizinstudium 2020
}

\author{
Lorenzo Käser \\ Universitätsspital Zürich
}

Mit Beginn des Herbstsemesters 2020 finden beachtliche Veränderungen in der Medizinstudium-Landschaft der Schweiz statt. Daher wollen wir zu diesem Zeitpunkt speziell jene Interessierten in der Ärzteschaft mit einer breiten, übersichtlichen Darstellung der Neuerungen und Entwicklungen informieren, die die junge Generation bei der Studiumwahl beraten möchten, besonders aber jene, die als Lehrärztinnen und Lehrärzte im Medizinstudium engagiert sind.

Die Medizinstudium-Standorte wurden eingeladen, in einer Eigendarstellung ihr Curriculum zu präsentieren. Die Übersichtstabelle im ersten Beitrag soll die wichtigsten Kenndaten vergleichen helfen und fasst die InternetLinks zusammen. Einzelne Lücken bei den Eigendarstellungen sind teils Corona geschuldet, teils verweisen die Studienorte stattdessen auf ihre Internet-Studiuminformationen.

\section{Pensionierungen, PROFILES und Profilierung}

Drei hauptsächliche Treiber der Umwälzungen und Entwicklungen im Medizinstudium in der Schweiz sind $\mathrm{zu}$ nennen: Die Zahl insbesondere von Grundversorgern, die altersbedingt für die Patientenversorgung in absehbarer Zeit nicht mehr zur Verfügung stehen werden, soll durch mehr Absolventen ausgeglichen werden. Ausserdem dämmerte die Erkenntnis, dass es für ein Land nicht ratsam ist, die Gesundheitsversorgung mit einem zu grossen Anteil ausländischer Fachkräfte sicherstellen zu wollen. Daher haben Bundesrat und Parlament das Sonderprogramm «Erhöhung der Anzahl Abschlüsse in Humanmedizin» lanciert. Die auf den ersten Blick beachtlichen Mittel des Bundes dafür haben in den universitären Hochschulen ohne medizinische Fakultät zu grossartigen Fantasien geführt. Schliesslich resultierten gut-schweizerische Kompromisse und Kooperationen, denen die politische Prägung teilweise anzusehen ist. Ob das Ziel erreicht werden kann, mit einer Produktionssteigerung von Medizinstudium-Absolvierenden die Lücken in der Gesundheitsversorgung zu kompensieren, bleibt offen, wie es auch im prägnanten Beitrag von Johann Steurer diskutiert wird.

Ein zweiter Faktor für die Veränderungen war der 2017 in Kraft gesetzte neue Lernzielkatalog für das Medizin- studium in der Schweiz. Während der frühere «Swiss Catalogue of Learning Objectives» (SCLO) der 2000er Jahre an einer eigentlich völlig unakademischen Listung von zu beherrschenden Krankheitsbildern, Fertigkeiten und klinischen Fachdisziplin-Begriffen krankte, steht beim neuen Katalog das unbestreitbar vernünftige Ausbildungsziel im Zentrum, welche Fähigkeiten mit welcher Selbständigkeit ein Assistenzarzt oder eine Assistenzärztin am ersten Tag der Weiterbildungsphase beherrschen muss. Das KatalogAkronym «PROFILES» (Principal Relevant Objectives and Framework for Integrative Learning and Education in Switzerland) beschäftigt seither die Beteiligten aller Stufen in der ganzen Schweiz. Insbesondere die Überprüfbarkeit der Fertigkeiten erzwingt zahlreiche Anpassungen bei der Vermittlung der Inhalte, aber auch bei den Examen. Das zeitgeistig-inflationäre «Assessment» von allem und jedem darf dabei aber keinesfalls zum Selbstzweck werden und nicht zulasten der verfügbaren Ausbildungszeit und Dozierendenleistung gehen.

Zum dritten bewirkten beide geänderten Rahmenbedingungen - grössere Studierendenzahlen mit neuen Anbietern sowie neue Lernziele - Reformmassnahmen an den Studienstandorten, wie Curriculumrevisionen, Kooperationen und eine stärkere Profilierung des eigenen Studiengangs. Dass sich künftige Medizinstudierende anhand des curricularen Angebots und des Profils einer Hochschule für einen Studiumstandort entscheiden, mag für die Schweiz noch ungewohnt sein, ist aber keine schlechte Entwicklung.

Corona schliesslich hat im ersten Halbjahr 2020 gezeigt, was die Chancen und Grenzen der Digitalisierung in der Medizinerausbildung sind. Doch auch in der klinischen Medizin hat die Digitalisierung schon längst Einzug gehalten und wird Berufsbild und Berufsausübung massiv verändern.

\section{Lorenzo Käser}

\author{
Dr. med. Lorenzo Käser \\ Ressort Lehre USZ \\ Direktion Forschung und Lehre \\ Universitätsspital Zürich \\ Rämistrasse 100 \\ 8091 Zürich
}

lorenzo.kaeser@usz.ch 